\title{
Uncontrolled Generator Operation of Interior PM Synchronous Machines Following High-Speed Inverter Shutdown
}

\author{
Thomas M. Jahns, Fellow, IEEE, and Vahe Caliskan, Student Member, IEEE
}

\begin{abstract}
Interior permanent magnet (IPM) synchronous machine drives are vulnerable to a special fault mode when gating is suddenly removed from the inverter switches during highspeed operation. The resulting IPM machine operation as a generator in combination with an uncontrolled rectifier must be properly understood and accounted for in the machine design to avoid damage to either the machine or inverter. An approximate closed-form solution is derived in this paper which relates the resulting machine phase current (and torque) to the IPM machine parameters, the dc-link voltage, and the rotor speed. The resulting operating characteristics are particularly interesting for IPM machines that have been designed with inductance saliency ratios greater than 2 (i.e., high-saliency machines). The validity of the approximate solution is confirmed using dynamic simulation results, and the implications of these results for the machine designer seeking to minimize or eliminate the impact of this undesired operating mode are thoroughly discussed.
\end{abstract}

Index Terms - Generator, interior permanent magnet synchronous machine, inverter shutdown.

\section{INTRODUCTION}

$\mathbf{T}$ HERE IS A variety of electric machine drive applications which require the machine to deliver a wide range of constant-power operation either as a motor or generator. Examples include high-performance machine tool spindle drives (motoring operation) and automotive alternators (generating operation). Interior permanent magnet (IPM) synchronous machines have been investigated for several years as appealing candidates for such applications [1]-[7]. It has been shown that, by properly adjusting the combination of magnetic saliency and permanent magnet flux in the IPM machine design, the resulting constant-power speed ratio (CPSR) can reach values of 10 or higher [7]. Such high values of CPSR are challenging for any type of ac (brushless) machine to achieve without compromising the machine and/or converter design.

Paper IPCSD 99-10, presented at the 1998 Industry Applications Society Annual Meeting, St. Louis, MO, October 12-16, and approved for publication in the IEEE TRANSACTIONS ON INDUSTRY APPLICATIONS by the Electric Machines Committee of the IEEE Industry Applications Society. This work was supported by the MIT/Industry Consortium on Advanced Automotive Electrical/Electronic Components and Systems. Manuscript released for publication February 15, 1999.

T. M. Jahns was with Massachusetts Institute of Technology, Cambridge, MA 02139-4307 USA. He is now with the Department of Electrical and Computer Engineering, University of Wisconsin, Madison, WI 53706-1691 USA (e-mail: t.jahns@ieee.org).

V. Caliskan is with the Department of Electrical Engineering and Computer Science, Massachusetts Institute of Technology, Cambridge, MA 02139-4307 USA (e-mail: vahe@mit.edu).

Publisher Item Identifier S 0093-9994(99)07485-X.

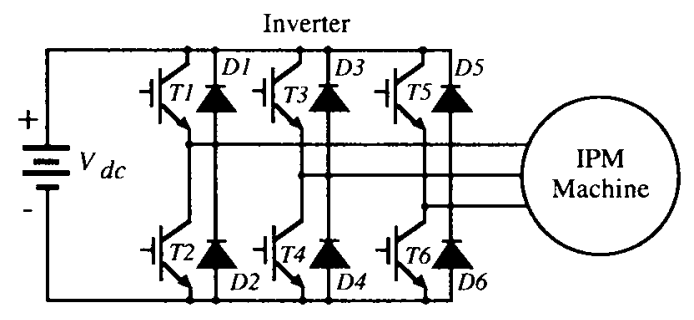

Fig. 1. Basic IPM machine drive configuration.

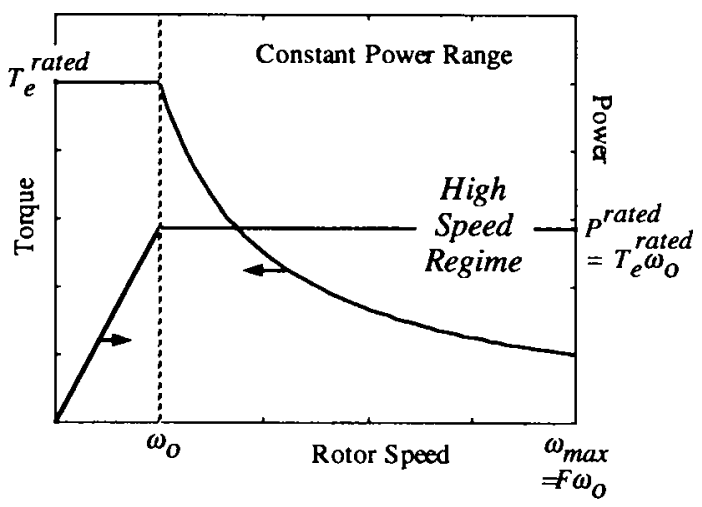

Fig. 2. IPM machine drive operating regimes.

However, there are several factors which must be taken into consideration when designing an IPM machine drive for a practical application requiring a wide CPSR. Consider a typical IPM motor drive configuration utilizing a classic six-switch full-bridge inverter (using insulated gate bipolar transistors (IGBT's), MOSFET's, or any other type of actively controlled switch) to excite the three-phase IPM machine as shown in Fig. 1. In this drive configuration, the amplitude of the lineto-line back EMF generated by the spinning IPM rotor magnets may significantly exceed the dc-link source voltage $V_{\mathrm{dc}}$ when the machine is rotating at high speeds. As shown in Fig. 2, "high speed" corresponds to the operating regime where the machine shaft speed considerably exceeds the corner speed $\omega_{0}$, which marks the lower threshold of the constant-power speed range. Such high-speed operation poses no problem as long as the inverter switches are operating properly in their controlled flux-weakening mode, since the maximum machine terminal voltage is automatically limited by the applied dc-link voltage $V_{\mathrm{dc}}$ [2].

Unfortunately, the situation changes considerably if a fault arises under these high-speed operating conditions which 
causes the inverter to "shut down" at high speeds, suddenly removing the gate excitation from all of the controlled inverter switches (see Fig. 1). Even though this shutdown mode is nondestructive in the sense that it creates no inverter shootthrough paths, the high amplitude of the machine back-EMF source causes current (and power) to flow back through the inverter freewheeling diodes to the dc link until the rotor speed is reduced sufficiently to extinguish the current flow.

Regardless of the machine's operating mode before the shutdown, the IPM machine acts as a generator immediately following the initiation of this shutdown mode with the inverter reduced to behaving as a simple full-bridge uncontrolled rectifier. It will be assumed throughout this paper that the dc link is capable of absorbing all of the power generated by the machine, protecting the inverter switches and diodes from exposure to dangerous overvoltages. Since the inverter transistor switches are totally inactive during this mode of operation, the amplitude of the resulting current and power flow is determined solely by the combination of IPM machine parameters, the instantaneous rotor speed, and the dc-link voltage $V_{\mathrm{dc}}$. The IPM machine shaft experiences a braking torque, since the machine is operating as a generator in this mode.

Depending on the IPM machine parameters and the operating conditions, the resulting shutdown mode current and power flow can be substantial during this undesired - but occasionally unavoidable-operating regime. The existence of this special fault mode consisting of high-speed uncontrolled generator (UCG) operation has been known to exist for some time, and some of the characteristics of this operating regime have been investigated in [8]. However, some of the most interesting performance characteristics and machine parameter dependencies associated with the UCG operating mode could not be readily observed using the dynamic simulation tools adopted in this earlier paper.

The purpose of this paper is to present a closed-form analysis of the UCG operating mode for the IPM machine drive which clearly exposes the key dependencies of the resulting phase current amplitudes on the machine parameters and the operating speed. The details of this analysis are presented in Section II, including extensions to account for the impact of magnetic saturation. Some the analytical results are counterintuitive at first sight, owing to the peculiar characteristics of the IPM machine in comparison to classic wound-field synchronous machines. Dynamic simulations are presented in Section III to confirm the closed-form results and to illustrate transient effects associated with this shutdown mode. The implications of these results for the machine drive designer seeking to achieve wide constant-power operating ranges are discussed in Section IV.

\section{ANALYSIS}

\section{A. Assumptions}

Three important assumptions are made in order to carry out this simplified analysis of high-speed UCG mode operation following inverter shutdown. In particular, the following points are assumed.

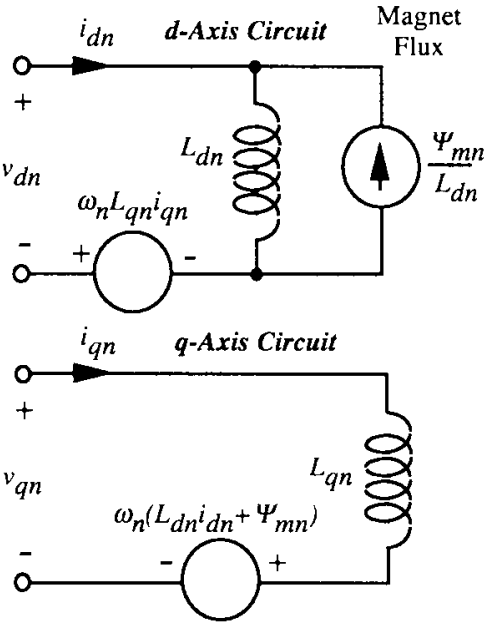

Fig. 3. IPM machine $d q$ equivalent circuits (normalized).

- IPM machine phase currents continue to be sinusoidal (i.e., continuous and harmonic free) during such operation, despite the fact that the uncontrolled rectifier applies nonsinusoidal voltage waveforms to the machine terminals.

- The rectifier acts to force the resulting phase currents to be strictly in phase with the fundamental components of the corresponding machine terminal phase voltages (or, more precisely, strictly out of phase by $180^{\circ}$ using motoring conventions for current polarity).

- Machine stator resistances and iron losses are sufficiently small to be ignored.

The validity of the first two assumptions are strongly supported by the results of the dynamic simulations which are presented in Section III, confirming that the IPM machine effectively filters the fifth, seventh, and higher harmonics which appear in the six-step voltage excitation waveforms during high-speed UCG mode operation. The basis for the second assumption regarding voltage and current phase alignment comes from the fundamental operating characteristics of any ac rectifier circuit. The validity of the third assumption actually rests on the performance characteristics of the particular IPM machine being investigated, but several references are available [9], [10] which confirm the feasibility of designing high-efficiency IPM machines consistent with this assumption.

\section{B. Machine Model and Normalization}

On the basis of these assumptions, steady-state analysis of IPM machine during high-speed UCG mode operation can be conveniently carried out in the synchronously rotating $d q$ reference frame. The interested reader is referred to [11] for more information about the derivation of the $d q$ models for the IPM synchronous machine. Fig. 3 presents the resulting $d$ - and $q$ axes equivalent circuits for an IPM machine which incorporate the convention that the $d$ axis is aligned with the magnetization axis of the rotor permanent magnets. Consistent with the third assumption presented above, the machine equivalent circuits in Fig. 3 include no resistive elements in the absence of copper and iron losses. Both $d$ - and $q$-axes stator currents are defined 
as positive when flowing into the machine, corresponding to motoring operation.

Since the IPM machine is a type of salient-pole synchronous machine, each of the $d$ - and $q$-axes equivalent circuits is characterized by a different value of stator inductance, $L_{d}$ and $L_{q}$, respectively. One of the features that sets the IPM machine apart from classic wound-field salient-pole synchronous machines is the fact that the inductance of the $d$ axis in the IPM machine is less than that of the orthogonal $q$ axis because of the low magnetic permeability of the permanent magnets $\left(\mu_{r} \approx 1\right)$. It is this reversed inequality which is ultimately responsible for the counterintuitive results which will be presented later in this paper.

All of the analysis presented in this paper uses normalized values of the machine parameters and the resulting voltage, current, and torque values. The subscript $n$ associated with each of these parameters and variables (see Fig. 3) signifies normalized values. The normalization scheme adopted in this paper was proposed in [12] and since then adopted by others [9] as a means of reducing the number of independent normalized parameters for the IPM machine from three to two. Base current and voltage are defined in rather conventional ways, corresponding to the rated peak phase values available from the inverter. For example, the base voltage $V_{o}$ is defined to be the peak phase-to-neutral fundamental-component voltage which can be applied to the wye-connected machine by the inverter, leading to the following relationship:

$$
V_{o}=\frac{2}{\pi} V_{\mathrm{dc}}
$$

According to this normalization scheme, base speed $\omega_{0}$ equals the value of rotor angular velocity (in electrical $\mathrm{rad} / \mathrm{s}$ ) at which the machine develops 1-p.u. voltage at its terminals with 1-p.u. phase current (i.e., rated voltage and rated current), with the phase current vector oriented to deliver maximum torque per ampere. Although the details will not be presented here [9], the net effect of this normalization is to cause the three normalized IPM machine parameters appearing in Fig. $3\left(L_{d n}\right.$, $L_{q n}$, and $\Psi_{m n}$ ) to be interrelated so that only two of these three parameters are independent. Derivative base values for this normalization scheme include the base flux $\psi_{o}=V_{o} / \omega_{o}$ and base torque $T_{e o}=(3 p / 2)\left(V_{o} I_{o} / \omega_{o}\right)$, where $p$ is the number of machine pole pairs.

\section{Steady-State Analysis}

Consistent with the IPM machine equivalent circuits presented in Fig. 3, the defining expressions for the steady-state $d$ - and $q$-axes stator voltages are

$$
\begin{aligned}
& V_{q n}=\omega_{n} \lambda_{d n}=\omega_{n} \Psi_{m n}+\omega_{n} L_{d n} I_{d n} \\
& V_{d n}=-\omega_{n} \lambda_{q n}=-\omega_{n} L_{q n} I_{q n}
\end{aligned}
$$

where uppercase variables signify steady-state values.

The phasor diagram shown in Fig. 4 corresponds to an IPM machine in the synchronously rotating $d q$ reference frame operating in the UCG mode. Note that the rectifier forces the machine voltage and current vectors $\left(\vec{V}_{s n}\right.$ and $\left.\vec{I}_{s n}\right)$ to be exactly $180^{\circ}$ out of phase, consistent with the assumptions

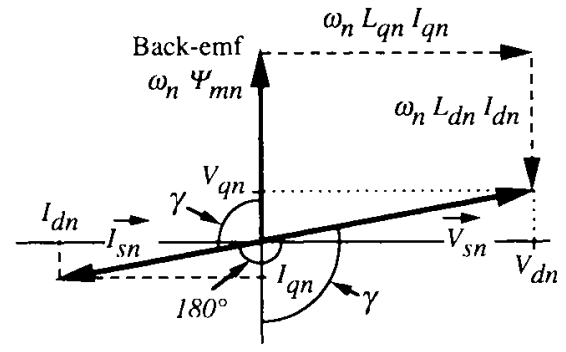

Fig. 4. Phasor diagram of IPM machine during UCG mode operation.

discussed above. It is also very interesting that the amplitude of the back EMF $\omega_{n} \Psi_{m n}$ is less than that of the stator voltage $V_{s n}$, an unusual condition made possible only by the fact that $L_{q n}$ is greater than $L_{d n}$ in the IPM machine. As indicated in Fig. 4, the largest portion of the resulting stator current consists of negative $d$-axis current, opposing the magnetization polarity of the permanent magnets.

Defining the angle separating the current vector $\vec{I}_{s n}$ from the positive $q$ axis as $\gamma$, the $d q$ components of $\vec{V}_{s n}$ and $\vec{I}_{s n}$ can be expressed as follows:

$$
\begin{array}{lr}
I_{d n}=-I_{s n} \sin \gamma & V_{d n}=V_{s n} \sin \gamma \\
I_{q n}=I_{s n} \cos \gamma & V_{q n}=-V_{s n} \cos \gamma .
\end{array}
$$

The expressions for $V_{d n}$ and $I_{q n}$ from (4) and (5) can be substituted into (3) to yield

$$
I_{s n}=\frac{-V_{s n} \sin \gamma}{\omega_{n} L_{q n} \cos \gamma} .
$$

This expression for the stator current amplitude can then be substituted into (2) and rearranged, leading to the following quadratic equation for $\cos \gamma$ :

$V_{s n}\left(L_{q n}-L_{d n}\right) \cos ^{2} \gamma+\omega_{n} \Psi_{m n} L_{q n} \cos \gamma+V_{s n} L_{d n}=0$.

At this point, it is useful to define two new variables as follows:

$$
\begin{aligned}
\xi & =\frac{L_{q n}}{L_{d n}} \\
\alpha & =\frac{\omega_{n} \Psi_{m n}}{V_{s n}}
\end{aligned}
$$

where $\xi$ corresponds to the inductance saliency ratio and $\alpha$ is the ratio of the back-EMF voltage at a given speed $\omega_{n}$ to the applied stator voltage. Special attention is drawn to the variable $\alpha$ which will be used frequently in this paper as a special normalization of the rotor speed appropriate for an investigation of UCG mode operation. In particular, note that $\alpha=1$ when the speed reaches the critical threshold value such that the back-EMF amplitude just equals the applied stator voltage. For an IPM machine designed with a normalized magnet flux of $\Psi_{m n}$ and excited with rated terminal voltage $\left(V_{s n}=1\right.$ p.u.), this threshold condition occurs when $\omega_{n}=$ $1 / \Psi_{m n}$ p.u.

Equation (7) can be rewritten using (8) and (9) in a more compact form as follows:

$$
(\xi-1) \cos ^{2} \gamma+\alpha \xi \cos \gamma+1=0
$$


which can be readily solved for $\cos \gamma$ to yield

$$
\cos \gamma=\frac{-\alpha \xi+\sqrt{(\alpha \xi)^{2}-4(\xi-1)}}{2(\xi-1)}
$$

for

$$
\alpha \geq \frac{2 \sqrt{(\xi-1)}}{\xi}, \quad \text { if } \xi>2 ; \quad \alpha \geq 1, \quad \text { if } \quad \xi \leq 2
$$

\section{Steady-State Results}

Equations (6) and (11) contain the key results from the preceding steady-state analysis, expressing the stator current which flows during UCG mode operation, subject to the important speed threshold condition in (12). These equations can be readily supplemented by the familiar equation for IPM machine torque [2] developed during this mode of operation, rearranged as follows:

$$
\begin{aligned}
T_{e n} & =\Psi_{m n} I_{q n}-\left(L_{q n}-L_{d n}\right) I_{q n} I_{d n} \\
& =I_{s n} \cos \gamma\left[\Psi_{m n}+L_{d n}(\xi-1) I_{s n} \sin \gamma\right] .
\end{aligned}
$$

The best way to gain some useful initial insights into the meaning of these expressions is to look at a specific example. Parameters for a 7.5-kW axially laminated IPM machine fabricated and tested by Soong [9] will be used to illustrate several of the key results in this paper. This machine is particularly interesting, since the design was adjusted to yield near-optimal flux-weakening capabilities, confirmed by a measured fluxweakening range exceeding $7.5: 1$. Key normalized parameters of this machine are

$$
\begin{aligned}
L_{d n} & =0.1994 \quad L_{q n}=1.336 \quad \xi=6.7 \\
\Psi_{m n} & =0.1986 .
\end{aligned}
$$

More details about this machine are provided in the Appendix. The fact that the normalized values of $L_{d n}$ and $\Psi_{m n}$ are nearly identical reflects the key machine design requirement for optimal flux weakening [3], [7]. It should be noted that the parameters given in (15) and (16) are for the unsaturated machine; the impact of saturation will be discussed later in this paper.

Fig. 5 shows a plot of the calculated stator current amplitude (in p.u.) during UCG mode operation as a function of the normalized rotor speed variable $\alpha$. The amplitude of the stator terminal voltage $V_{s n}$ used in (6) is 1 p.u. throughout this operating mode as set by the uncontrolled rectifier. Fig. 6 shows the corresponding calculated torque (also normalized) as a function of $\alpha$.

Figs. 5 and 6 begin to reveal some of the intriguing characteristics exhibited by the IPM machine operating in this uncontrolled rectifier mode. Intuitively, one might expect that the machine phase current flowing through the rectifier would be zero when the back-EMF amplitude just equals the reflected dc-link voltage $(\alpha=1)$. In fact, Fig. 5 indicates that a substantial phase current flows when $\alpha=1$. Even more interesting and counterintuitive, the curve indicates that this current continues to flow, even when the speed falls below the critical $\alpha=1$ condition, so that the back-EMF voltage

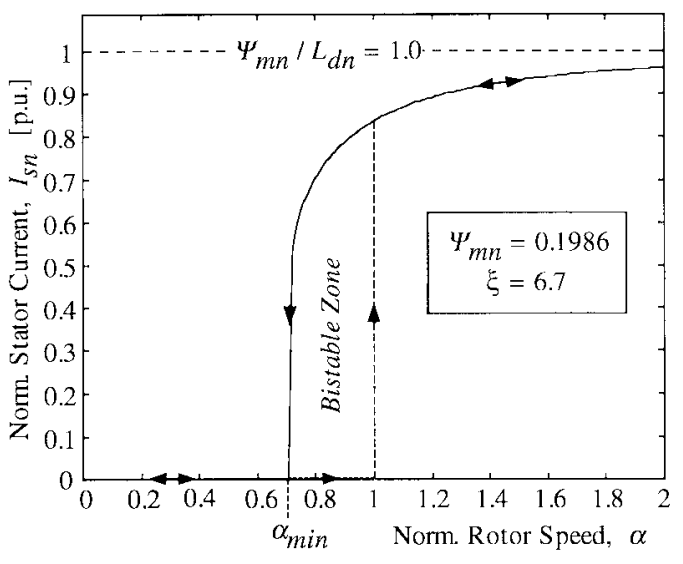

Fig. 5. Calculated normalized stator current during UCG mode operation as a function of normalized rotor speed $\alpha$.

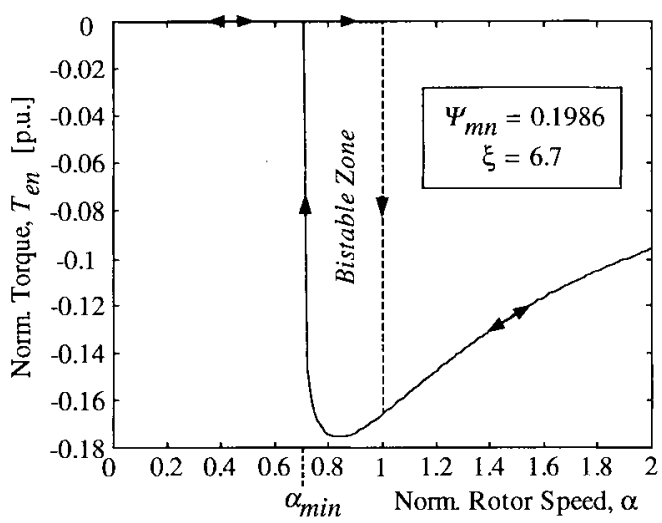

Fig. 6. Calculated normalized torque during UCG mode operation as a function of normalized rotor speed $\alpha$.

is less than the reflected dc-link voltage. The current finally extinguishes when the speed drops to the minimum value of $\alpha$ satisfying (12), which will be henceforth referred to as $\alpha_{\min }$.

Before leaving the stator current curve in Fig. 5, it should be noted that the stator current appears to asymptote to a value of 1 p.u. for high values of speed. That is, the steady-state stator current during UCG mode operation will not exceed its rated value (1 p.u.) for this particular machine, suggesting that the inverter diodes will not be exposed to dangerous overcurrents. Manipulation of (6) and (11) leads to the following interesting result:

$$
\lim _{\alpha \rightarrow \infty} I_{s n}=\frac{\Psi_{m n}}{L_{d n}} .
$$

Thus, the particular asymptotic behavior observed in Fig. 5 is caused by selecting an IPM machine design such that $\Psi_{m n}=L_{d n}$ in order to optimize it for wide ranges of constant power operation. The high-speed stator current will asymptote to values greater or less than 1 p.u. for any nonoptimal machine design.

Surprising features are also found in the Fig. 6 torque curve, with peak braking (negative) torque developed at a value of $\alpha<1$. It is worth noting that rated torque is not identical to the base torque value because of the normalization scheme 


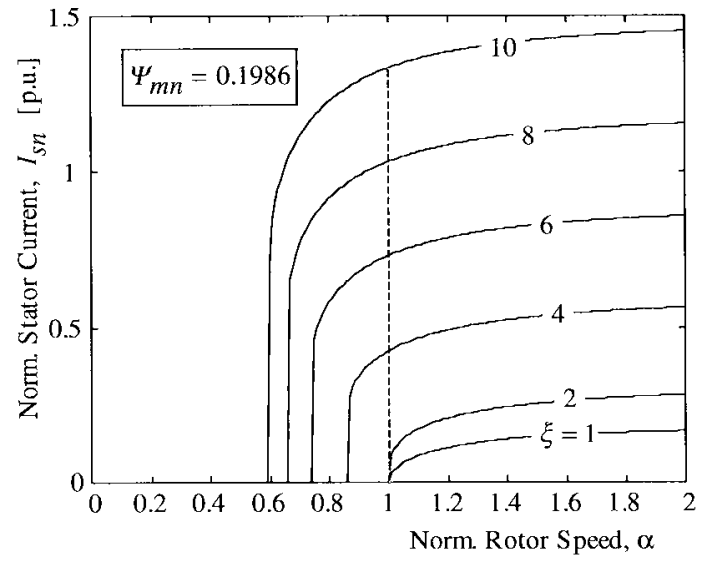

Fig. 7. Impact of inductance saliency ratio $\xi$ on current versus speed characteristics during UCG mode operation.

[9]. Rated torque $(53.1 \mathrm{~N} \cdot \mathrm{m})$ for this particular IPM machine corresponds to 0.70 p.u., meaning that the peak braking torque in Fig. 6 ( -0.175 p.u.) is actually $25 \%$ of the rated torque amplitude. Interestingly, the braking torque amplitude diminishes as the speed is increased above the $\alpha=1$ value.

Another intriguing aspect of Figs. 5 and 6 results which requires explanation is the unexpected bistable operation exhibited in the regime between $\alpha=\alpha_{\text {min }}$ and 1. Consider the IPM machine connected to an uncontrolled rectifier and initially spinning at a rotor speed less than $\alpha=\alpha_{\min }$ with no current flowing in the stator windings. If the rotor speed is gradually increased, no current will flow when the speed is between $\alpha_{\min }$ and 1 because the back EMF is less than the reflected dc-link voltage, causing the rectifier diodes to remain reverse biased and nonconducting. However, once the critical threshold rotor speed value of $\alpha=1$ is reached, the rectifier diodes become forward biased, and the preceding analysis predicts that the rectifier-machine system will almost instantly switch to an active conduction state with substantial stator current flow ( $I_{s n}$ is approximately 0.8 p.u. at $\alpha=1+$ ). This rectifier conduction state persists, even if the rotor speed is subsequently lowered below $\alpha=1$, with the current extinguishing only when the speed drops below the critical $\alpha_{\min }$ value. This unusual hysteretic behavior is noted on the curves in Figs. 5 and 6 by means of the directed arrows.

\section{E. Parameter Dependencies}

Equation (12) indicates that the value of $\alpha_{\min }$ monotonically decreases as the value of the inductance saliency ratio $\xi$ increases. Conversely, simple manipulation of (12) reveals that the unusual bistable operating region disappears entirely $\left(\alpha_{\min }=1\right)$ when the value of the saliency ratio is less than or equal to $2(\xi \leq 2)$. This includes all classic wound-field salient-pole synchronous machines, since these machines are characterized by saliency ratios less than one (i.e., $L_{q n}<$ $\left.L_{d n}\right)$.

The derivation carried out above for UCG mode operation applies to a salient-pole synchronous machine with any value of inductance saliency ratio, regardless of whether $\xi$ is less than or greater than unity. Figs. 7 and 8 show families of

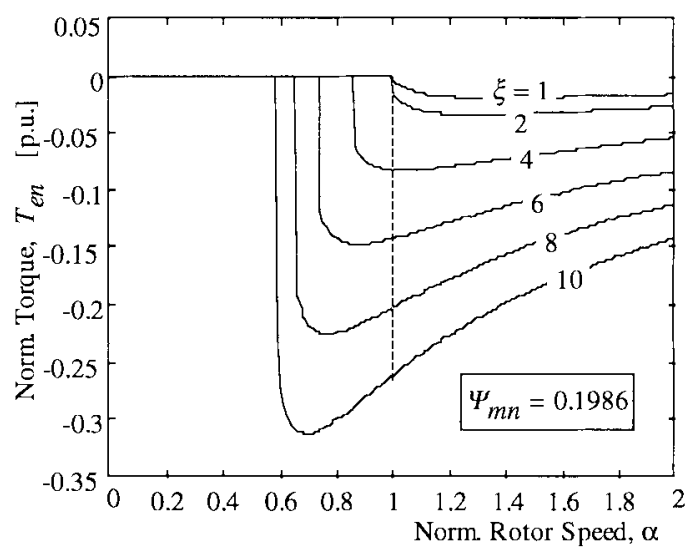

Fig. 8. Impact of inductance saliency ratio $\xi$ on torque versus rotor speed characteristics during UCG mode operation.

calculated steady-state curves for the stator current amplitude and torque, respectively, for several values of $\xi$ ranging from 1 to 10 . The value of the normalized magnet flux $\Psi_{m n}$ is held fixed at the same value (0.1986 p.u.) used in Figs. 5 and 6.

Just as predicted by (12), the curves in Figs. 7 and 8 indicate that the value of $\alpha_{\min }$ is 1 for inductance saliency ratios of 2 or less. In this regime, the stator current and the torque both increase monotonically with speed for $\alpha>1$, consistent with heuristic arguments suggesting that the current will grow as the back EMF increases above the critical dc-link voltage threshold value. On the other hand, Figs. 7 and 8 confirm that $\alpha_{\min }$ monotonically decreases as the saliency ratio increases above $\xi=2$, diminishing to a value of $\alpha_{\min }=0.6$ when the saliency ratio reaches 10 .

It should be noted that the $d$-axis inductance $L_{d n}$ is a dependent variable according to the chosen normalization scheme and its value decreases as the value of the saliency ratio $\xi$ increases for a given value of magnet flux $\psi_{m n}$. Consequently, the limiting normalized value of high-speed stator current $\left(=\Psi_{m n} / L_{d n}\right)$ gradually increases in Fig. 7 as the value of $\xi$ is raised for fixed $\Psi_{m n}$.

\section{F. Magnetic Saturation Effects}

It is well known by workers in the field that magnetic saturation can have a significant effect on the performance of IPM machines [7]. More specifically, the $q$-axis inductance $L_{q n}$ is particularly susceptible to magnetic saturation at elevated values of $q$-axis current. Such drops in the value of $L_{q n}$ are undesirable because of their negative impact on machine torque production and other performance characteristics. In contrast, the $d$-axis inductance $L_{d n}$ is typically almost free of saturation effects because its value is dominated by the rotor magnet slots which behave as large air gaps in the $d$-axis magnetic circuit.

A straightforward approach adopted in this work for including magnetic saturation in the preceding analysis of UCG mode operation is to make $L_{q n}$ an analytic function of the $q$-axis current $I_{q n}$. This approach has been shown by others to yield relatively accurate results [7], although accuracy can be further increased if desired by using more complicated models 


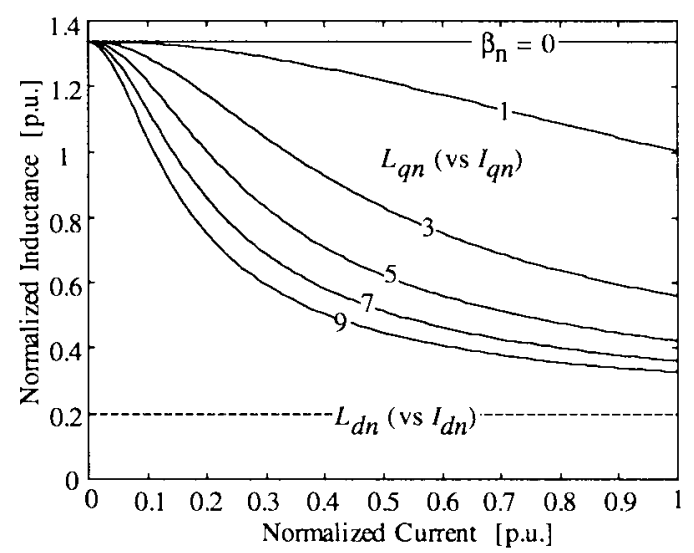

Fig. 9. Models of $L_{q n}$ versus $I_{q n}$ and $L_{d n}$ versus $I_{d n}$, for varying levels of magnetic saturation parameter $\beta_{n}$.

which account for cross-coupling effects between the two axes [13].

After studying some of the documented saturation curves for $L_{q n}$ collected by Soong [7], a nonlinear model for $L_{q n}$ as a function of $I_{q n}$ was selected as an effective approximation

$$
L_{q n}=L_{d n}+\frac{\left(L_{q n o}-L_{d n}\right)}{\left[1+\left(\beta_{n} I_{q n}\right)^{2}\right]^{1 / 2}}
$$

where $L_{q n o}$ is the unsaturated value of $L_{q n}$ when $I_{q n}$ is zero, and model parameter $\beta_{n}$ determines whether and to what degree $L_{q n}$ is vulnerable to saturation as $I_{q n}$ increases $\left(\beta_{n}=0\right.$ for no saturation effect).

This model has the attractive feature of smoothly and gradually transitioning into saturation as the current amplitude is raised without any steps in the derivative. Fig. 9 shows how the saturation characteristics of $L_{q n}$ vary as $\beta_{n}$ is increased using the unsaturated inductance values for the $7.5-\mathrm{kW}$ IPM machine described in the Appendix.

The basic machine electrical equations presented earlier as (2) and (3) can now be solved using (18) to represent $L_{q n}$ as a function of $I_{q n}$. Unfortunately, the nonlinearities present in (18) make it impractical to develop a closed-form solution for $I_{s n}$ in the presence of saturation. However, the nonlinearity is well behaved, so that the equations can be readily solved by iteration. For example, the calculations leading to Figs. 5 and 6 have been redone for the same IPM machine using the values of saturation parameter $\beta_{n}$ and accompanying saturation curves shown in Fig. 9. While $L_{q n}$ is a function of $I_{q n}$, it is assumed that the value of $L_{d n}$ is constant, unaffected by saturation.

The resulting family of steady-state current and torque curves in Figs. 10 and 11 illustrate the progressive impact of increasing levels of magnetic saturation. These curves demonstrate that increasing the saturation factor $\beta_{n}$ has much the same effect as reducing the machine's inductance saliency ratio $\xi$. That is, the speed threshold value for current conduction $\alpha_{\min }$ gradually increases toward 1 as $\beta_{n}$ is increased to high values, although the asymptotic high-speed current value given in (17) does not change, since neither $\Psi_{m n}$ nor $L_{d n}$ is affected by saturation according to this model.

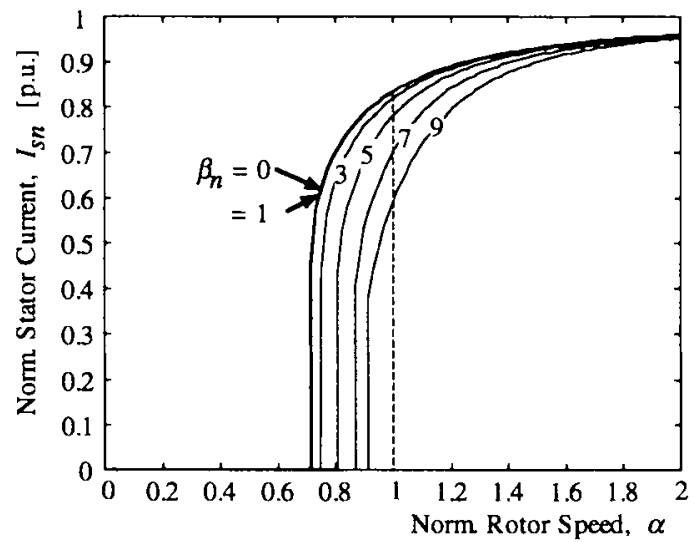

Fig. 10. Impact of saturation parameter $\beta_{n}$ on current versus rotor speed characteristics during UCG mode operation.

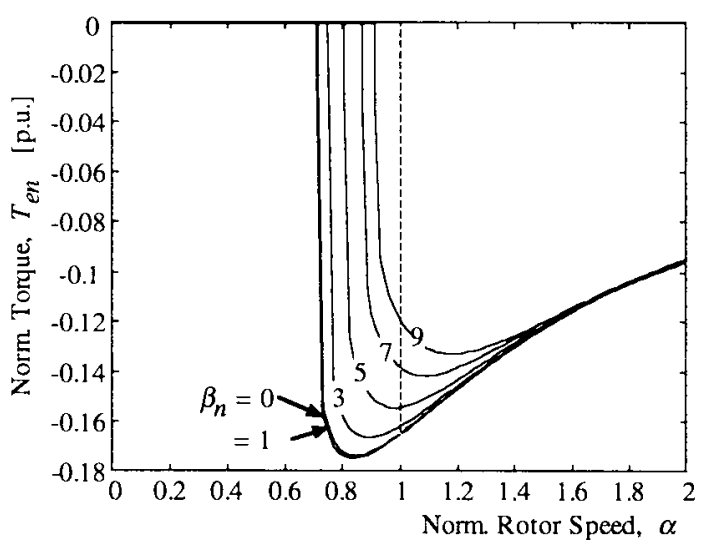

Fig. 11. Impact of saturation parameter $\beta_{n}$ on torque versus rotor speed characteristics during UCG mode operation.

One interesting observation is that the stator current amplitude at a given rotor speed $\alpha$ actually decreases as the value of $L_{q n}$ is reduced via saturation, yielding another counterintuitive trend caused by the IPM machine's unique $L_{q n}>L_{d n}$ inductance relationship. The torque amplitudes in Fig. 11 gradually diminish as a result of increasing saturation levels, which is consistent with expectations for the effect of reduced saliency ratios on torque production [see (14)].

\section{DyNAMic Simulation}

As a means of providing preliminary verification for the results presented in the previous section, a dynamic simulation of the IPM machine-rectifier combination was prepared using the Saber simulation language. The IPM machine was modeled in the $d q$ synchronously rotating reference frame using the dynamic state equations which can be readily extracted from the two-axes equivalent circuit presented earlier in Fig. 3. The machine model used in this simulation is lossless, just as in the steady-state analysis described in the preceding section.

Parameters for the same 7.5-kW IPM machine described in the Appendix and summarized in (15) and (16) were used in the simulations. Magnetic saturation was included by using the same basic model described by (18), with the value of the $q$-axis inductance $L_{q n}$ dependent on the instantaneous value 
(a)

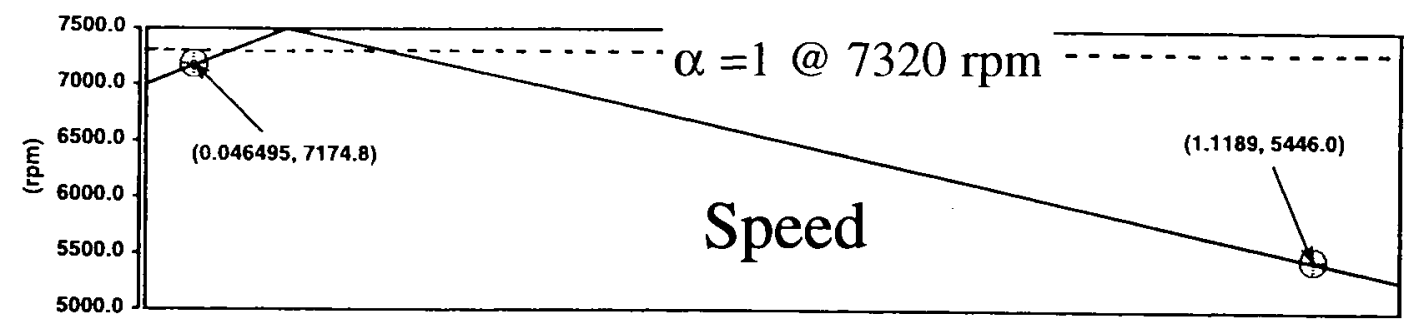

(b)

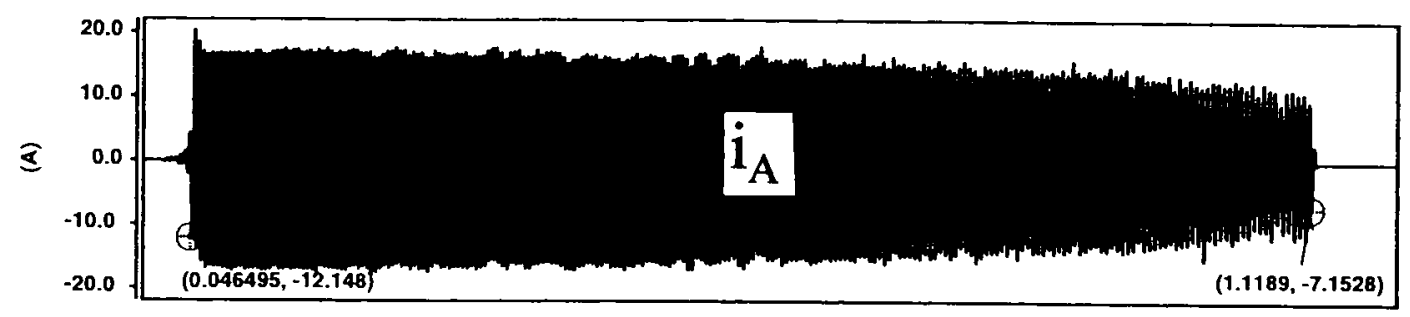

(c)

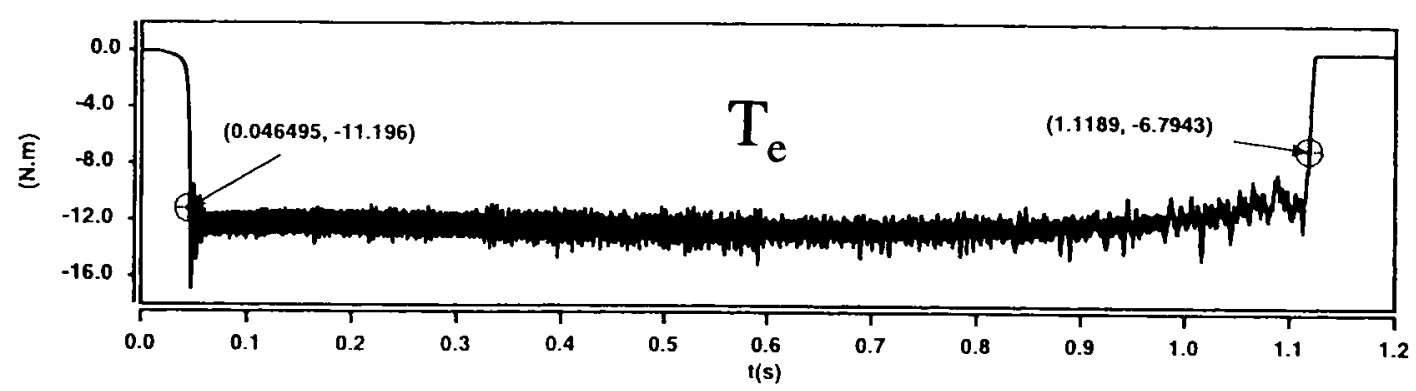

Fig. 12. Simulation of UCG mode operation in response to speed ramp around $\alpha=1$. (a) Speed. (b) $i_{A}$. (c) $T_{e}$.

of the $q$-axis current $i_{q n}$. The value of $\beta_{n}$ was adjusted to approximate the relatively modest level of saturation measured in the actual machine. More specifically, a value of $\beta_{n}=$ 1.085 was selected so that the value of $L_{q}$ drops from its unsaturated value of 80.4 to $58 \mathrm{mH}$ when $i_{q}$ reaches rated current $\left(=20.5 \mathrm{~A}=1\right.$ p.u.). Allowing $L_{q n}$ to vary as a function of $i_{q n}$ makes it necessary to introduce an additional voltage term into the $q$-axis dynamic equation taking the form $\left(\delta L_{q n} / \delta i_{q n}\right) \cdot i_{q n} \cdot\left(d i_{q n} / d t\right)$.

An interesting set of simulation results is presented in Fig. 12 for an applied rotor speed ramp shown as Fig. 12(a). The critical condition of $\alpha=1$ when the back-EMF amplitude just equals the reflected dc-link voltage corresponds to 7320 $\mathrm{r} / \mathrm{min}$ for this machine. The speed ramp has been adjusted so that the speed begins below this threshold, but eventually exceeds it, reaching a maximum value of $7500 \mathrm{r} / \mathrm{min}(\alpha=$ 1.025) before ramping downward. The simulation is initiated with no stator current flowing, and the speed derivative is sufficiently low so that the machine operates under quasisteady-state conditions in its UCG mode.

Inspection of the Fig. 12 waveforms confirms that the IPM machine exhibits the predicted bistable behavior illustrated in Figs. 5 and 6 during UCG mode operation. That is, the stator current ignites rather suddenly in the vicinity of $\alpha=1$ and continues to flow until the speed is reduced sufficiently to the point $\left(\alpha_{\min }\right)$ that current conduction can no longer be sustained. The amplitudes of the stator current and braking torque which are developed in the dynamic simulation agree very closely with the calculated steady-state values from (6) and (14).
Predictions by the steady-state analysis of the rotor speeds at which the IPM machine transitions into and out of UCG mode differ from the values extracted from the dynamic simulation waveforms in Fig. 12 by approximately $2 \%-3 \%$. Since space does not permit a thorough discussion of these transition phenomena in this paper, it must suffice here to note that the simplifying assumptions described earlier which make the closed-form analysis possible are responsible for contributing these modest errors.

Fig. 13 provides an expanded view of the current ignition transient, including the corresponding line-to-neutral phase voltage in addition to the current. The impact of this sudden transition is very apparent in both waveforms. Note that the phase voltage converts from a nearly sinusoidal waveshape set by the machine's open-circuit back-EMF voltage source into the classic six-step waveform set by the dc-link voltage source.

Given the bistable nature of the operating speed regime between $\alpha=\alpha_{\min }$ and $\alpha=1$, an important question arises as to what happens if the IPM machine is operating under inverter excitation as a motor in this speed regime when the inverter suddenly shuts down. Will the currents decay to zero, or will the machine switch immediately into UCG mode operation? Dynamic simulations of this real-world situation indicate that the answer depends on the preshutdown operating conditions.

For example, Fig. 14 shows waveforms for the $7.5-\mathrm{kW}$ IPM machine spinning at $6500 \mathrm{r} / \mathrm{min}(\alpha=0.89)$ when the machine is operating under motoring conditions with $i_{q}=15 \mathrm{~A}=0.73$ p.u. and $i_{d}=-10 \mathrm{~A}=-0.49$ p.u. prior to the inverter shutdown at $t=0$. These waveforms indicate that the IPM machine immediately transitions into UCG mode operation 
(a)

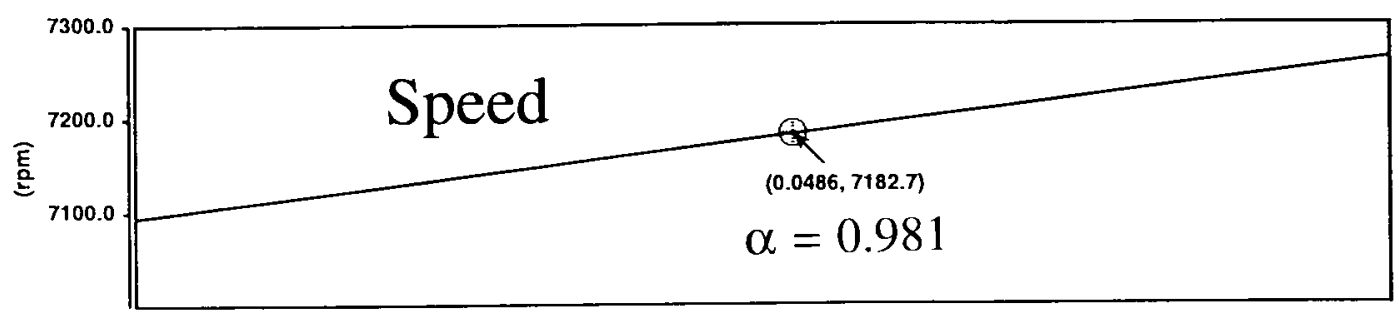

(b)

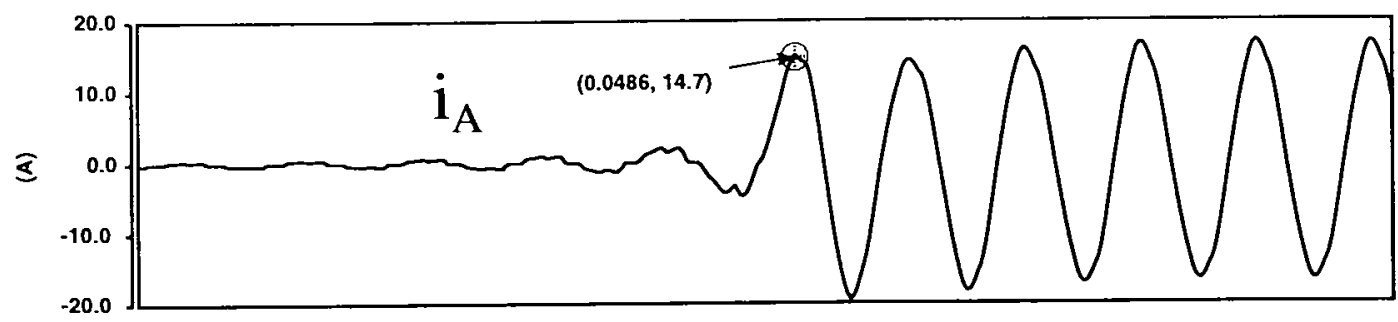

(c)

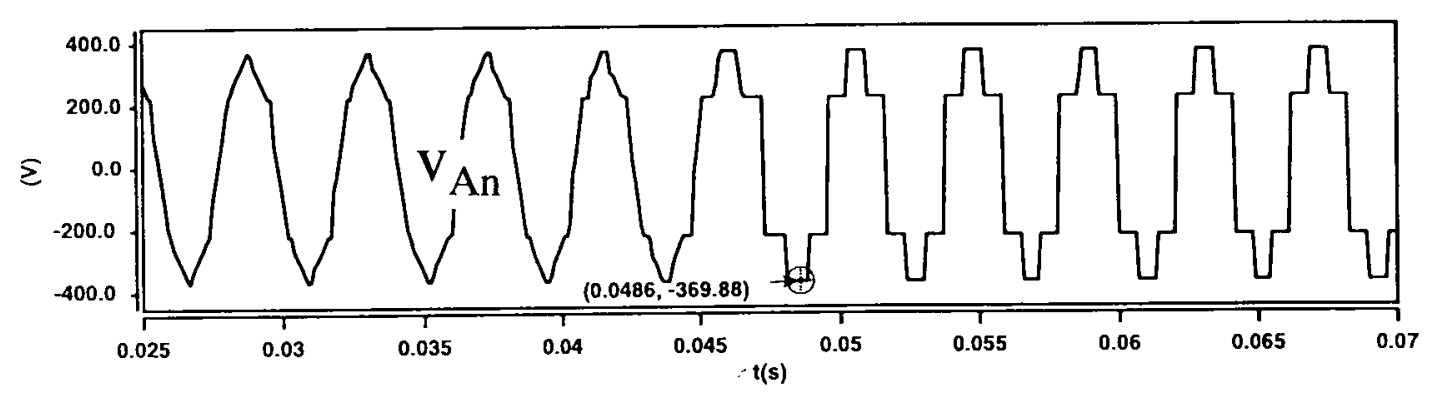

Fig. 13. Simulation of current ignition transient marking start of UCG mode operation. (a) Speed. (b) $i_{A}$. (c) $v_{A n}$.

(a)

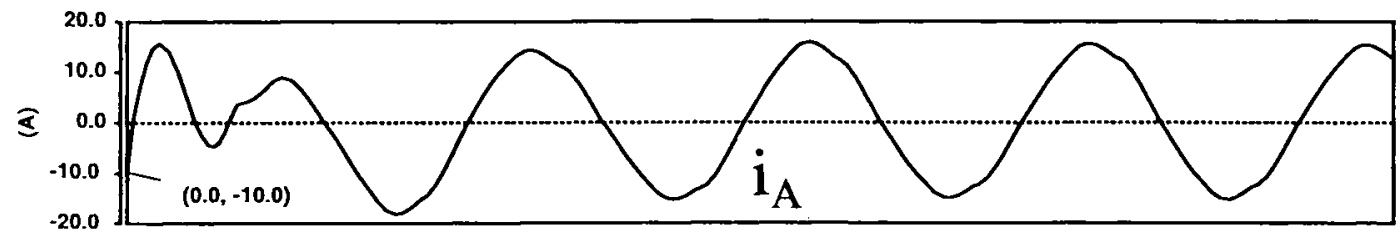

(b)

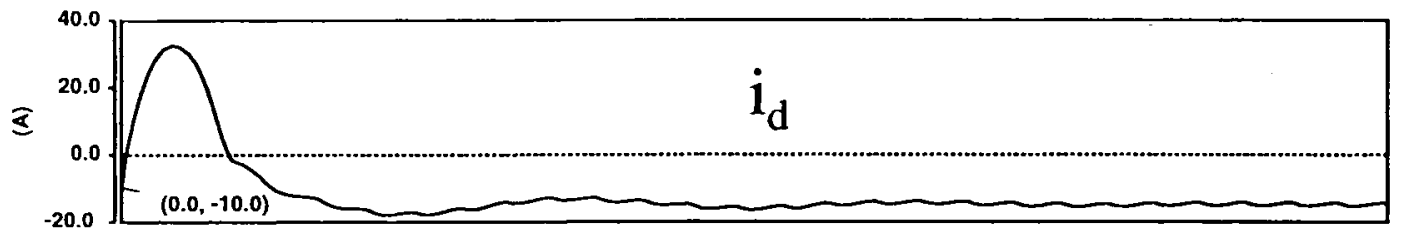

(c)

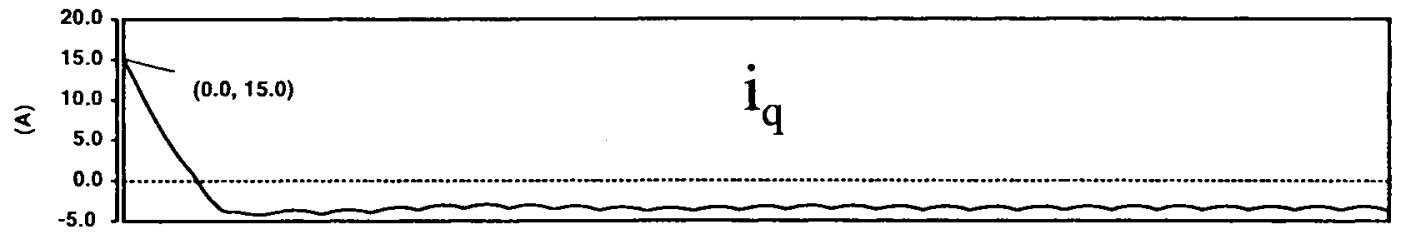

(d)

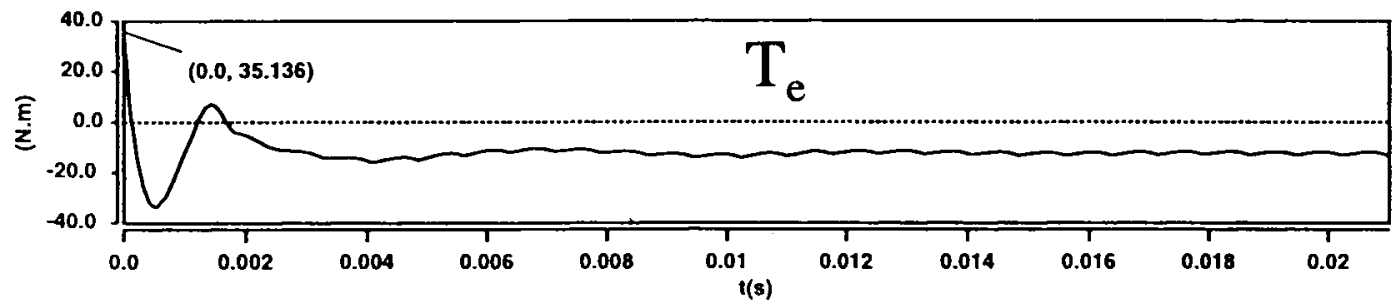

Fig. 14. Simulation of UCG mode initiation triggered by shutdown during motoring. (a) $i_{A}$. (b) $i_{d}$. (c) $i_{q}$. (d) $T_{e}$.

following an initial transient lasting only about $5 \mathrm{~ms}$. On the other hand, another simulation (not shown) for identical conditions except with less preshutdown current $\left(i_{q}=5 \mathrm{~A}\right.$
$=0.24$ p.u.; $i_{d}=-3 \mathrm{~A}=-0.15$ p.u.) shows that the currents immediately decay to zero and stay there unless the speed is subsequently raised above $\alpha=1$. A complete mapping from 
initial current conditions to postshutdown operating mode has not been carried out, but it is clear that the IPM machine is vulnerable to transitioning into the UCG mode following unexpected inverter shutdowns when the motor speed $\alpha$ is between $\alpha_{\min }$ and 1 .

A final significant observation drawn from the Fig. 14 transient waveforms and other cases not shown here is that the transient response of $i_{d}$ never takes this current to large negative values which are demagnetizing in nature. In fact, the initial transient for $i_{d}$ is in the positive direction, reaching approximately +1.5 p.u. for the Fig. 14 case. Following this positive transient, $i_{d}$ settles smoothly to its steady-state generator mode value with minimal additional transient excursions in the negative direction. Although it is dangerous to draw broad conclusions based on limited data, there are hopeful signs that the shutdown transient may not generally pose a significant threat of demagnetizing the rotor magnets.

\section{DESIGN IMPLiCATIONS}

There are several important observations which can be drawn from this work which have noteworthy implications for the design of IPM machines, particularly for cases where wide ranges of constant-power operation are desired.

\section{A. Postshutdown Current Amplitude}

The significance of the $\Psi_{m n} / L_{d n}=1$ p.u. condition as a prerequisite for achieving wide ranges of constant-power operation in IPM machines has been known for some time [3]. This paper further highlights the significance of this ratio as the limiting amplitude value of the steady-state machine phase current during UCG mode operation at high speeds. Designing the IPM machine with $\Psi_{m n} / L_{d n}$ ratios of 1 or less thus appears to be a good practice in order to protect the inverter flyback diodes from current values above 1 p.u. following unexpected inverter shutdowns at high speed when $\alpha$ may be much greater than 1. Since the largest proportion of this generator mode stator current flows as negative $d$-axis current, the design decision to limit $\psi_{m n} / L_{d n}$ design values to 1 or less also helps to reduce the demagnetization risk for the rotor magnets.

\section{B. Inductance Saliency Ratio $\xi$}

Generally, increasing the value of the inductance saliency ratio $\xi$ in an IPM machine is desirable as a means of reducing the amount of expensive magnet material needed to develop the desired level of machine torque. Considerable effort has been invested by many workers to find ways to increase this saliency [14]. However, the results of this paper suggest that increasing the saliency ratio $\xi$ has some less desirable side effects with regards to UCG mode operation. More specifically, increasing the value of $\xi$ reduces the value of $\alpha_{\min }$, meaning that the machine becomes vulnerable to transitioning into UCG mode operation following shutdowns at progressively lower speed. For example, an inductance saliency ratio of $\xi=10$ reduces the generator mode threshold value to $\alpha_{\min }=0.6$, which is $40 \%$ below the speed at which the back-EMF amplitude equals the reflected dc-link source voltage.

Furthermore, results presented earlier in this paper (see Figs. 7 and 8) suggest that increasing the value of the saliency ratio $\xi$ has the effect of increasing the generator mode current amplitude and the corresponding braking torque at a given rotor speed $\alpha$. These observations are not meant to suggest that a high value of saliency ratio $\xi$ is undesirable, but that there are performance ramifications and tradeoffs which should be considered when setting the saliency design value.

\section{Wide Constant-Power Operating Range}

When designing an IPM machine drive for an application requiring a wide range of constant-power operation, it would be highly desirable to design the system so that it is immune to UCG mode operation under all operating conditions if possible. This type of conservative approach to the drive system design imposes some very real restrictions on the selection of the IPM machine parameters.

The critical machine design constraint required to assure immunity to UCG mode operation comes from the requirement that the peak machine rotor speed must not exceed the critical $\alpha=\alpha_{\min }$ condition. If the required constant speed power ratio (CSPR) is $F$ where $F \geq 1$, the maximum normalized rotor speed is $F$ p.u. (see Fig. 2), which corresponds to a maximum $\alpha$ value of $\alpha=F \Psi_{m n}$ for rated terminal voltage $\left(V_{s n}=1\right.$ p.u.). Using (12), this leads to the following inequality:

$$
F \Psi_{m n} \leq \alpha_{\min }=\frac{2 \sqrt{(\xi-1)}}{\xi}, \quad \text { for } \xi \geq 2
$$

which can be easily rearranged to yield the following machine design constraint:

$$
\Psi_{m n} \leq \frac{2 \sqrt{(\xi-1)}}{F \xi}, \quad \text { for } \xi \geq 2 .
$$

As noted earlier in this paper, the normalization scheme has been chosen so that the IPM machine design can be defined using only the two independent machine parameters $\Psi_{m n}$ and $\xi$. Using these two parameters as the axes, the condition for immunity to UCG mode operation in (20) has been plotted as a family of dotted-line curves for different values of CPSR $F$ as shown in Fig. 15. The design space to the left of each curve represents all IPM machine designs which provide the desired immunity for a particular value of $F$. It must be noted that these curves do not include the effects of magnetic saturation which would have the effect of shifting them to the right toward $\Psi_{m n} \leq 1 / F$ for increasing levels of $L_{q n}$ saturation.

Fig. 15 also includes a solid line which represents the locus of all IPM machine designs which meet the critical condition for optimal flux weakening defined by $\Psi_{m n}=L_{d n}$ [7]. Thus, if the machine designer wishes to design an IPM machine that is optimized for wide ranges of constant power operation but immune to UCG mode operation, Fig. 15 indicates that the design task becomes increasingly more difficult as the desired speed ratio $F$ is increased, because the minimum required value of inductance saliency ratio $\xi$ progressively increases. For example, Fig. 15 indicates that designing such a machine 


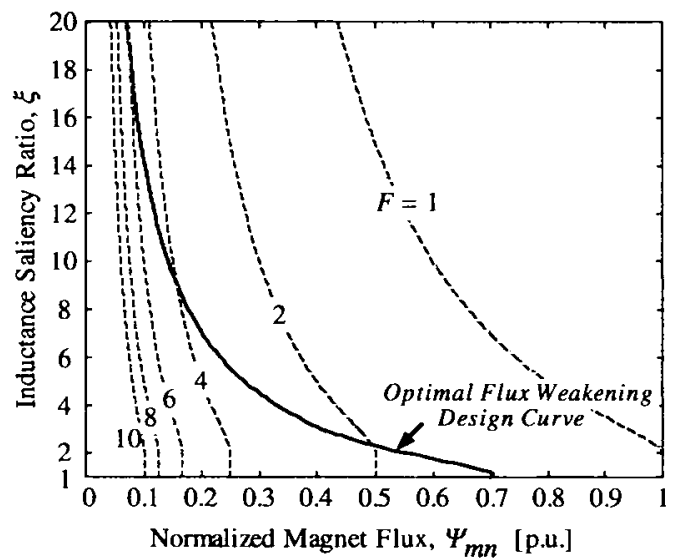

Fig. 15. IPM machine design space showing constraint for immunity to UCG mode as a function of constant-power speed ratio $F$.

with a CSPR of 4 requires that the inductance saliency ratio $\xi$ be approximately 9 or higher, which is not a minor design challenge.

Inclusion of realistic values of magnetic saturation will provide some relief in this constraint, but will not change the basic conclusion. As a result, one is led to the conclusion that total immunity to UCG mode operation is most practical to achieve for IPM machines when the desired value of CPSR $F$ is less than approximately 4-5. For higher values of $F$, it is still highly desirable to design the machine with the minimum practical value of magnet flux $\Psi_{m n}$ while meeting the optimum flux weakening criteria shown in Fig. 15, in order to approach the UCG immunity requirement as closely as possible.

\section{CONCLUSIONS}

This paper has attempted to illuminate the key performance characteristics of IPM machines during UCG mode operation following unexpected inverter shutdowns at high speed. The most interesting results developed during this study are caused by that fact that the $L_{q} / L_{d}$ inductance saliency ratio for an IPM machine is greater than 1 , in direct contrast to classic wound-rotor salient-pole synchronous machines. Key results of this work include the following.

- IPM machines with $L_{q} / L_{d}$ ratios greater than 2 are vulnerable to operating in the UCG mode whenever the rotor speed is above a minimum threshold value which is less than the speed at which the line-to-line machine back EMF equals the reflected dc-link voltage.

- If $L_{q} / L_{d}$ is greater than 2 , there is a limited speed range below the speed at which the back EMF equals the reflected dc-link voltage in which the machine can exhibit bistable steady-state behavior, operating either in the open-circuit mode with zero stator current, or in the UCG mode with potentially significant current and braking torque.

- The amplitude of the stator current during UCG mode operation at high speeds depends heavily on the ratio of the permanent magnet flux to the $d$-axis inductance
$\Psi_{m} / L_{d}$, with the majority of this current flowing as negative (demagnetizing) $d$-axis current.

- Designing an IPM for optimal flux-weakening operation ( $\Psi_{m} / L_{d}=$ rated current) and total immunity to UCG mode operation places constraints on the design, forcing it to low values of magnet flux and high saliency ratios. These constraints get progressively more difficult to meet as the desired CPSR increases.

Future work is planned to conduct experimental tests to verify key analytical results.

\section{APPENDIX}

The 7.5-kW IPM machine which has been discussed in this paper is an axially laminated machine which was designed, built, and tested by Dr. W. Soong [9]. Key electrical parameters of the machine and associated inverter drive include the following:

$$
\begin{array}{rlrl}
L_{q} & =80.4 \mathrm{mH} \text { (unsat) } & & L_{d}=12.0 \mathrm{mH} \\
\Psi_{m} & =0.245 \mathrm{~Wb}(\mathrm{pk} l-n) & & T_{e}^{\mathrm{rated}}=53.1 \mathrm{~N} \cdot \mathrm{m} \\
V_{\mathrm{dc}} & =590 \mathrm{~V} & & I_{o}=20.5 \mathrm{~A}(\mathrm{pk}) \\
\omega_{o} & =304.4 \mathrm{rad}^{e} / \mathrm{s}=1453 \mathrm{r} / \mathrm{min} \quad(4 \text { poles }) .
\end{array}
$$

\section{REFERENCES}

[1] B. Sneyers, D. W. Novotny, and T. A. Lipo, "Field-weakening in buried permanent magnet AC motor drives," IEEE Trans. Ind. Applicat., vol. IA-21, pp. 398-407, Mar./Apr. 1985.

[2] T. M. Jahns, "Flux-weakening regime operation of an interior permanent magnet synchronous motor drive," IEEE Trans. Ind. Applicat., vol. IA-23, pp. 681-689, July/Aug. 1987.

[3] R. F. Schiferl and T. A. Lipo, "Power capability of salient pole permanent magnet synchronous motor in variable speed drive applications," IEEE Trans. Ind. Applicat., vol. 26, pp. 115-123, Jan./Feb. 1990.

[4] A. K. Adnanes and T. M. Undeland, "Optimum torque performance in PMSM drives above rated speed," in Conf. Rec. IEEE-IAS Annu. Meeting, Oct. 1991, pp. 169-175.

[5] S. Morimoto, Y. Takeda, T. Hirasa, and K. Taniguchi, "Expansion of operating limits for permanent magnet motor by current vector control considering inverter capacity," IEEE Trans. Ind. Applicat., vol. 26, pp. 866-871, Sept./Oct. 1990.

[6] A. Fratta, A. Vagati, and F. Villata, "Design criteria of an IPM machine suitable for field-weakened operation," in Proc. Int. Conf. Electric Machines, 1990, pp. 1059-1064

[7] W. Soong and T. J. E. Miller, "Field-weakening performance of brushless synchronous AC motor drives," Proc. Inst. Elect. Eng.-Elect. Power Applicat., vol. 141, no. 6, pp. 331-339, 1994.

[8] A. Adnanes, R. Nilssen, and R. Rad, "Power feed-back during controller failure in inverter fed permanent magnet synchronous motor drives with flux weakening," in Proc. IEEE PESC'92, 1992, pp. 958-963.

[9] W. Soong, "Design and modeling of axially-laminated interior permanent magnet motor drives for field-weakening applications," Ph.D. dissertation, Dep. Electron. Elect. Eng., Univ. Glasgow, Glasgow, U.K., Sept. 1993.

[10] R. D. King, "ETX-II 70 hp electric drive system performance-Component tests," in Proc. 10th Int. Electric Vehicle Symp., 1990, pp. 878-887.

[11] P. C. Krause, O. Wasynczuk, and S. D. Sudhoff, Analysis of Electric Machinery. Piscataway, NJ: IEEE Press, 1995, pp. 499-534.

[12] A. K. Adnanes, "Torque analysis of permanent magnet synchronous motors," in Proc. IEEE PESC'91, 1991, pp. 695-701.

[13] A. Vagati, M. Pastorelli, and G. Franceschini, "Effect of magnetic crosscoupling in synchronous reluctance motors," in Proc. Intelligent Motion Conf., 1997, pp. 279-285.

[14] I. Boldea, Reluctance Synchronous Machines and Drives. Oxford, U.K.: Clarendon, 1996. 


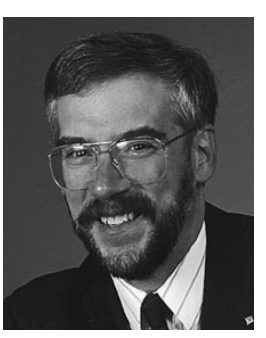

Thomas M. Jahns (S'73-M'79-SM'91-F'93) received the S.B. and S.M. degrees in 1974 and the Ph.D. degree in 1978 from Massachusetts Institute of Technology, Cambridge, MA, all in electrical engineering.

In October 1998, he joined the faculty of the University of Wisconsin, Madison, as a Professor in the Department of Electrical and Computer Engineering, where he is also an Associate Director of the Wisconsin Electric Machines and Power Electronics Consortium (WEMPEC). Prior to this, he was with GE Corporate Research and Development for 15 years, where he pursued new power electronics and motor drive technology in a variety of research and management positions. His research interests have included permanent magnet synchronous machines for a variety of applications ranging from high-performance machine tools to low-cost appliance drives. During 1996-1998, he conducted a research sabbatical at Massachusetts Institute of Technology, where he directed research activities in the area of advanced automotive electrical systems and accessories as Codirector of an industrysponsored automotive consortium.

Dr. Jahns was recognized as a Distinguished Lecturer by the IEEE Industry Applications Society (IAS) during 1994-1995 and by the IEEE Power Electronics Society (PES) during 1998-1999. He has served two terms as President of the PES (1995-1996) and has been a Member of the IAS Executive Board since 1992

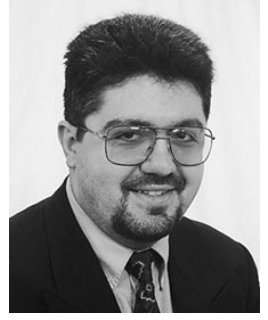

Vahe Caliskan (S'88) was born in Istanbul, Turkey, in 1968. He received the B.S. (with honors) and the M.S. degrees in electrical engineering from the University of Illinois, Chicago, in 1990 and 1993 , respectively. $\mathrm{He}$ is currently working toward the $\mathrm{Ph} . \mathrm{D}$. degree in electrical engineering at Massachusetts Institute of Technology, Cambridge.

From 1990 to 1994, he was a Research Assistant in the Power Electronics Research Laboratory and a Teaching Assistant in the Electrical Engineering and Computer Science Department, University of Illinois, Chicago. During the summer of 1992, he was with Philips Laboratories, Briarcliff Manor, NY. He is currently a Research Assistant in the Massachusetts Institute of Technology Laboratory for Electromagnetic and Electronic Systems, where he is involved in the development of dual-voltage automotive electrical power systems. His research interests include power electronics, analog circuit design, and computer-aided modeling/simulation.

Mr. Caliskan is a member of Sigma Xi, Tau Beta Pi, and Eta Kappa Nu. 